\title{
Caspase-14 is an anti-apoptotic protein targeting apoptosis-inducing factor in lung adenocarcinomas
}

\author{
HSIN-YUAN FANG ${ }^{1,2}$, CHIH-YI CHEN $^{2}$, MIN-FA HUNG $^{4}$, YI-TING HSIAO ${ }^{4}$, TING-CHIEH CHIANG ${ }^{4}$, \\ TZE-YI LIN ${ }^{3}$, HUI-WEN CHANG ${ }^{3}$, KUAN-CHIH CHOW $^{4}$ and WEN-JE KO ${ }^{1}$ \\ ${ }^{1}$ Graduate Institute of Clinical Medicine, National Taiwan University School of Medicine, Taipei; ${ }^{2}$ College \\ of Medicine, China Medical University and Division of Thoracic Surgery, Department of Surgery and \\ ${ }^{3}$ Department of Pathology, China Medical University Hospital, China Medical University, Taichung; ${ }^{4}$ Graduate \\ Institute of Biomedical Sciences, National Chung Hsing University, Taichung, Taiwan, R.O.C.
}

Received January 11, 2011; Accepted March 28, 2011

DOI: 10.3892/or.2011.1292

\begin{abstract}
Using apoptosis-inducing factor protein as bait in a yeast hybrid assay to screen protein libraries, we identified three proteins that interacted with apoptosis-inducing factor: human homolog of yeast Rad23 protein A (hHR23A), microsomal glutathione $\mathrm{S}$-transferase and caspase-14 (casp-14). In this study, we investigated the expression and function of casp-14 in lung adenocarcinomas (LADC). Our results showed that monoclonal antibodies were specific to casp-14, and that casp-14 was highly expressed in LADC. Casp-14 overexpression correlated with tumor stage, cell differentiation and lymphovascular involvement, suggesting that casp-14 was associated with tumor cell growth and metastatic potential. In vitro, casp-14 interacted with apoptosis-inducing factor, and silencing of casp-14 expression reduced cisplatin resistance. Our data suggest that casp-14 is an anti-apoptotic protein targeting apoptosis-inducing factor and increases cisplatin resistance in LADC cells.
\end{abstract}

Correspondence to: Dr Kuan-Chih Chow, Graduate Institute of Biomedical Sciences, National Chung Hsing University, Taichung, Taiwan, R.O.C

E-mail: kcchow@dragon.nchu.edu.tw

Dr Wen-Je Ko, Graduate Institute of Clinical Medicine, National Taiwan University School of Medicine, Taipei, Taiwan, R.O.C E-mail: kowj@ntu.edu.tw

Abbreviations: AIF, apoptosis-inducing factor protein; CPD, cyclobutane pyrimidine dimmers; EGFP, enhanced green fluorescent protein; GST, glutathione S-transferase; hHR23, human homolog of yeast Rad23 protein; LADC, lung adenocarcinoma; NTLT, non-tumor lung tissues; PCD, programmed cell death; UV, ultraviolet

Key words: caspase-14, apoptosis-inducing factor, cisplatin resistance, lung adenocarcinoma, anti-apoptosis

\section{Introduction}

Programmed cell death (PCD) is a controlled process of tissue demolition that is imperative for regulating the development and tissue homeostasis in multicellular organisms (1). Based on morphological and biochemical characteristics three types of PCD have been categorized (2-4). However, only type I PCD, which is featured by DNA fragmentation, chromatin condensation, and appearance of micronuclei (nuclear events), as well as phospholipid scrambling, extensive membrane blebbing, loss of matrix attachment and cellular shrinkage (cytoplasmic events), is mediated by apoptogenic proteins, such as apoptosis-inducing factor (AIF), cytochrome $\mathrm{c}$ and caspases (5). AIF and cytochrome $\mathrm{c}$ are located in intermembranous space (IMS) of mitochondria. PCD, which is initiated by cytochrome c release and activation of caspases, is called caspase-dependent apoptosis. PCD, which is initiated by nuclear translocation of AIF, but does not require caspase activation, on the other hand, is called caspase-independent apoptosis. Type II PCD and type III PCD are also initiated in a caspase-independent manner (6).

Besides being an apoptogenic protein during programmed cell death, AIF is in fact an indispensable mitochondrial flavoprotein (7). Upon exposure to genotoxic agents, e.g., cisplatin, adriamycin, staurosporine, camptothecin and etoposide, AIF is rapidly released from mitochondria and translocated to the nucleus to initiate degradation of poly(ADP-ribose) polymerase, DNA fragmentation, chromatin condensation and formation of micronuclei, the hallmarks of nuclear apoptosis (7-12). However, AIF does not interact with karyopherins, proteins involved in nucleocytoplasmic shuttling (13). We therefore, propose that nuclear translocation of the AIF may require vehicles other than karyopherins. Using fulllength AIF protein as bait in a yeast hybrid assay to search libraries containing cytoplasmic and nuclear proteins (Level Biotechnology Inc., Taipei, Taiwan), three proteins that interacted with AIF: human homolog of yeast $\operatorname{Rad} 23$ protein A (hHR23A) in the nuclear protein library, and hHR23A, microsomal glutathione S-transferase and casp-14 in the cytoplasmic protein library, were identified (Table I). As the 
Table I. Proteins that interact with AIF in libraries containing cytoplasmic (Modules LPap002, LPap003 and LPap005, Level Biotechnology Inc., Taipei, Taiwan) or nuclear (Module LPtf010) proteins, which are identified by using AIF as the bait in a yeast two-hybrid assay.

\begin{tabular}{|c|c|c|c|c|c|c|c|}
\hline \multirow[b]{2}{*}{ Module } & \multirow[b]{2}{*}{ Location } & \multirow[b]{2}{*}{ Accession no. } & \multirow[b]{2}{*}{ Gene name } & \multicolumn{2}{|c|}{ Growth selection } & \multicolumn{2}{|c|}{ X-gal assay } \\
\hline & & & & $1 \mathrm{st}$ & Repeat & $1 \mathrm{st}$ & Repeat \\
\hline \multicolumn{8}{|c|}{ Cytoplasmic } \\
\hline LPtf005 & E8, E9 & NM_005053 & Rad23 (S. cerevisiae) homolog A & +++ & +++ & +++ & +++ \\
\hline LPtf005 & $\mathrm{F} 2, \mathrm{~F} 3$ & NM_020300 & Microsomal glutathione S-transferase 1 (MGST1) & +++ & +++ & +++ & +++ \\
\hline LPtf005 & F10, F11 & NM_012114 & Caspase-14 & +++ & +++ & +++ & +++ \\
\hline \multicolumn{8}{|l|}{ Nuclear } \\
\hline LPtf010 & E10, E11 & NM_005053 & $\operatorname{Rad} 23$ (S. cerevisiae) homolog A & \multicolumn{2}{|c|}{+++} & \multicolumn{2}{|c|}{+++} \\
\hline
\end{tabular}

shortest member of the caspase family, casp-14 is phylogenetically closest to casp-2 (shared $26 \%$ amino acid identity). However, according to pathophysiological functions, casp-14 is not able to be categorized into any of the three groups: cytokine activators, initiator or executioner caspases. Unlike the other caspases, casp-14 is not proteolytically processed at Asp residue in the linker region $(1,14)$, but is cleaved between ${ }^{152}$ Ile and ${ }^{153}$ Lys in terminally differentiated keratinocytes (15). Interestingly, knockout of casp-14 gene increases epidermal storage of profilaggrin and sensitivity to ultraviolet (UV) B, suggesting that casp-14 plays a role in protein maturation of filaggrin (16), and possibly in DNA repair. Overexpression of casp-14 has been detected in several of epithelial malignancies, indicating that casp-14 could play a role in carcinogenesis and disease progression of cancers $(17,18)$. However, casp-14 has not been studied in lung adenocarcinomas, of which the incidence and mortality have increased dramatically in the last two decades in Taiwan (19). Although cigarette smoking has been suggested being a key factor of disease progression and treatment failure (12), a portion of patients who do not respond well to the radiation- and chemotherapy in Taiwan are women and non-smokers (20). Treatment failures are major reasons for the high disease-related mortality. Because casp-14 binds AIF, we therefore hypothesize that casp-14 may act as an anti-apoptotic factor by interfering with AIF function and reducing drug sensitivity in cancer cells.

In this study, we examined the expression level of casp-14 in lung adenocarcinoma (LADC) cells and pathological specimens. The correlation between casp-14 expression and patient survival was evaluated statistically. Interaction between casp-14 and AIF as well as the effect of casp-14 on cell growth and drug resistance was characterized in vitro.

\section{Materials and methods}

Patients and tissue specimens. The patients in this study were from the same cohort used in a previous study (19). Briefly, from January to December 2001, sera and tissue specimens were collected from 264 patients with newly diagnosed NSCLC. Samples from all patients, for whom at least one follow-up examination or death was documented, were pathologically confirmed NSCLC. Of the 264 patients, 107 were diagnosed as having lung ADC. The stage of the disease was classified according to the new international staging system for lung cancer (21). The Medical Ethics Committee approved the protocol, and written informed consent was obtained from every patient before surgery. All patients had undergone surgical resection and radical N2 lymph node dissection. Tumor size, lymph node number, differentiation, vascular invasion and mitotic number were also evaluated. Patients with lymph node involvement or loco-regional recurrence received irradiation at the afflicted areas. Patients with distant metastasis were treated with chemotherapy. After treatment, patients were routinely followed every 3-6 months in the outpatient department. Results from blood examination, biochemical studies, chest radiography, abdominal sonography, whole body bone scan and computerized tomography scans of chest that indicated any evidence of disease were interpreted as tumor recurrence and metastasis. The average age of the male patients $(n=84)$ was $60.6 \pm 1.4$ years and that of the female patients $(n=23)$ was $53.3 \pm 1.3$ years. Immunohistochemical staining was carried out using a single-blind procedure.

Reverse transcription-polymerase chain reaction (RT-PCR). Following total RNA extraction and synthesis of the firststrand cDNA, an aliquot of cDNA was subjected to 35 cycles of PCR to determine the integrity of $\beta$-actin mRNA $(12,19)$. The cDNA used in the following RT-PCR was adjusted according to the quality and quantity of $\beta$-actin mRNA. The primer sequences were selected by a web program, Primer3 at http://frodo.wi.mit.edu/primer3. For caspase-14, the primers are: Casp-14s: 5'-GAAATGAGCAATCCGCGGTCT-3' [sense primer, nts 46-66, NM_012114] and Casp-14a: 5'-CTACTGCAGATACAGCCGTTT-3' (antisense primer, nts 777-757).

Preparation of mouse antibodies. DNA sequence corresponding to full-length casp-14 was amplified by primer sequences containing EcoRI (sense) and HindIII (antisense) restriction sites respectively. The primer sequences were Casp-14s: 5'-TCCGAATTCATGAGCAATCCGCGGTCTTTG-3' (EcoRI site is underlined) and Casp-14a: 5'-GCAAGCTT GCTACTGCAGATACAGCCGTTT-3' (HindIII site is under- 
lined). The restriction fragment of casp-14 was cloned into an expression vector $\mathrm{pET}-32 \mathrm{~b}^{+}$(Promega KK, Tokyo, Japan). Bacterial colony containing pET32+-casp-14 was selected, and induced by isopropyl- $\beta$-D-thiogalactopyranoside (IPTG) to mass-produce casp-14. Recombinant protein was purified by a nickel-affinity column. Affinity-purified casp-14 was used to immunize BALB/c mice, and sensitivity of antiserum $\left(\mathrm{OD}_{405}\right.$ $>0.3$ at 1:6,000 dilutions) was measured by enzyme-linked immunosorbent assay (ELISA). Specificity of antibodies was determined by the appearance of a $35-\mathrm{kDa}$ band in immunoblotting of lung cancer cell extract (22). Monoclonal antibodies were produced by a hybridoma technique, and casp-14-specific antibodies were screened by the methods mentioned above.

Immunoblotting analysis and immunocytochemistry. Total cell lysate was prepared by mixing $5 \times 10^{7}$ cells $/ 100 \mu \mathrm{l}$ phosphate-buffered saline with equal volume of $2 \mathrm{X}$ loading buffer (50 mM Tris, $\mathrm{pH} 6.8,150 \mathrm{mM} \mathrm{NaCl}, 1 \mathrm{mM}$ disodium EDTA, $1 \mathrm{mM}$ PMSF, $10 \%$ glycerol, $5 \% \beta$-mercaptoethanol, $0.01 \%$ bromophenol blue and 1\% SDS). Electrophoresis was carried out in a $10 \%$ polyacrylamide gel with $4.5 \%$ stacking. Proteins were transferred to a nitrocellulose membrane after electrophoresis. The membrane was probed with specific antibodies. The signal was amplified by biotin-labeled goat anti-mouse $\mathrm{IgG}$, and peroxidase-conjugated streptavidin. The protein was visualized by exposing the membrane to X-Omat film (Eastman Kodak, Rochester, NY) with enhanced chemiluminescent reagent (NEN, Boston, MA). Immunocytochemical staining was performed by an immunoperoxidase method using labeled streptavidin-biotin complex (Dako, LSAB2 System, Carpenteria, CA) $(12,19)$. For confocal immunofluorescence microscopy, the second antibodies contained fluorescence proteins.

Immunohistochemistry. Immunohistological staining was performed by an immunoperoxidase method $(12,19)$. Briefly, following removal of paraffin with xylene and absolute alcohol, antigen was retrieved by treating samples in boiling water for $10 \mathrm{~min}$. Endogenous myeloperoxidase was inactivated with $3 \% \mathrm{H}_{2} \mathrm{O}_{2}$ and $0.1 \%$ sodium azide at room temperature for $15 \mathrm{~min}$. After incubation with monoclonal antibodies (1:100 dilutions) specific to caspase-14 at room temperature for $2 \mathrm{~h}$, specimens were treated with biotin-conjugated goat anti-mouse immunoglobulin, and then peroxidase-conjugated streptavidin. Chromogenic development was in 3-amino9-ethylcarbazole. The slides were counterstained with $50 \%$ hematoxylin at RT for $45 \mathrm{sec}$ and the blue color was enhanced in running water for $20 \mathrm{~min}$. The crimson precipitates were identified as positive staining. The non-tumor counterpart of the LADC tissue served as a negative control, and a section of gelatin-embedded H23 LADC cells was used as a positive control for each run of immunostaining.

Slide evaluation. In each pathological section, non-tumor lung tissue (NTLT) served as an internal negative control. Slides were evaluated by two independent pathologists blinded to the clinicopathological knowledge. The ImmunoReactive Scoring system was adapted for this study (23). Briefly, a specimen was considered having strong signals when $>50 \%$ of cancer cells were positively stained; intermediate, if $25-50 \%$ of the cells stained positive; weak, if $<25 \%$ or $>10 \%$ of the cells were positively stained; and negative, if $<10 \%$ of the cells were positively stained. Cases with strong and intermediate casp-14 signals were classified as casp- $14^{+}$, and those with weak or negative casp-14 signals were classified as casp-14.

Cytotoxicity assay. Cells were seeded at 5,000 cells/well $18 \mathrm{~h}$ prior to challenge with various doses of cisplatin. The control group was treated with RPMI-1640. Following cell challenge for $48 \mathrm{~h}, 10 \mu \mathrm{l}$ of WST-1 (BioVision, Mountain View, CA) was added and continued incubation for $2 \mathrm{~h}$. Percent survival of cells was quantified by comparing the number of viable cells in the treatment group with that in the control group. All procedures were performed in triplicate. This assay measures both replicating and static cells (12).

Statistical analysis. The relationship between caspase-14 expression and clinicopathological parameters was analyzed by $\chi^{2}$ test. Survival curves were plotted using the KaplanMeier estimator (24). Statistical difference in survival among different groups was compared by the log-rank test (between Casp-14+ ${ }^{+}$and Casp-14- groups) (25). Statistical analysis was performed using GraphPad Prism5 statistical software (San Diego, CA). Statistical significance was set at p-value $<0.05$.

\section{Results}

Expression of caspase-14 in LADC cells determined by $R T-P C R$. Expression of caspase-14 was examined by RT-PCR in eight lung cancer cell lines. Caspase-14 was detected in all cell lines (Fig. 1A). Following sequence analysis (by Mission Biotech, www.missionbio.com.tw, Taipei, Taiwan, on ABI PRISM, model 3730), nucleotide sequence of cDNA fragments from the nine cell lines matched that of caspase-14: NM_012114, Homo sapiens caspase-14, apoptosis-related cysteine peptidase (caspase-14), mRNA, identities $=775 / 777(99.7 \%)$. Two point mutation sites were identified: $109 \mathrm{cta} \rightarrow 109 \mathrm{gta}$ (Leu21Val) in three of nine samples and 724aaa $\rightarrow 724 \mathrm{gaa}$ (Lys226Glu) in six of nine samples (The sequence was presented in GenBank, EU262664.1, and Fig. 1B). Interestingly, mutation at these two positions increased the probability of protein nuclear localization when protein sequences were analyzed by a PSORT II prediction program (http://psort.ims.u-tokyo. ac.jp/) (Table II). A bipartite nuclear localization signal (NLS) 'RRMAEAELVQEGKARKT' was identified between amino acid residues 211-237.

Characterization of monoclonal antibodies to caspase-14 and expression of caspase-14 in LADC cell lines. Specificity of monoclonal antibodies was determined by an immunoblotting of LADC cell lysate. A $35-\mathrm{kDa}$ protein corresponding to the anticipated molecular mass of caspase-14 was recognized (14). For confirming the specificity of antibodies, we inserted caspase-14 gene into a pcDNA3.1 vector (pcDNA3.1-casp-14) or a pEGFP-N1 vector, which carried an enhanced green fluorescent protein (EGFP) gene (pEGFP-casp-14). As shown in Fig. 2A, the monoclonal antibodies recognized the anticipated 


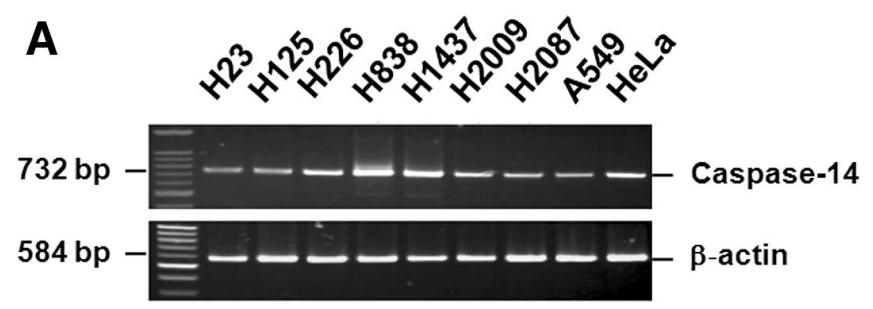

B

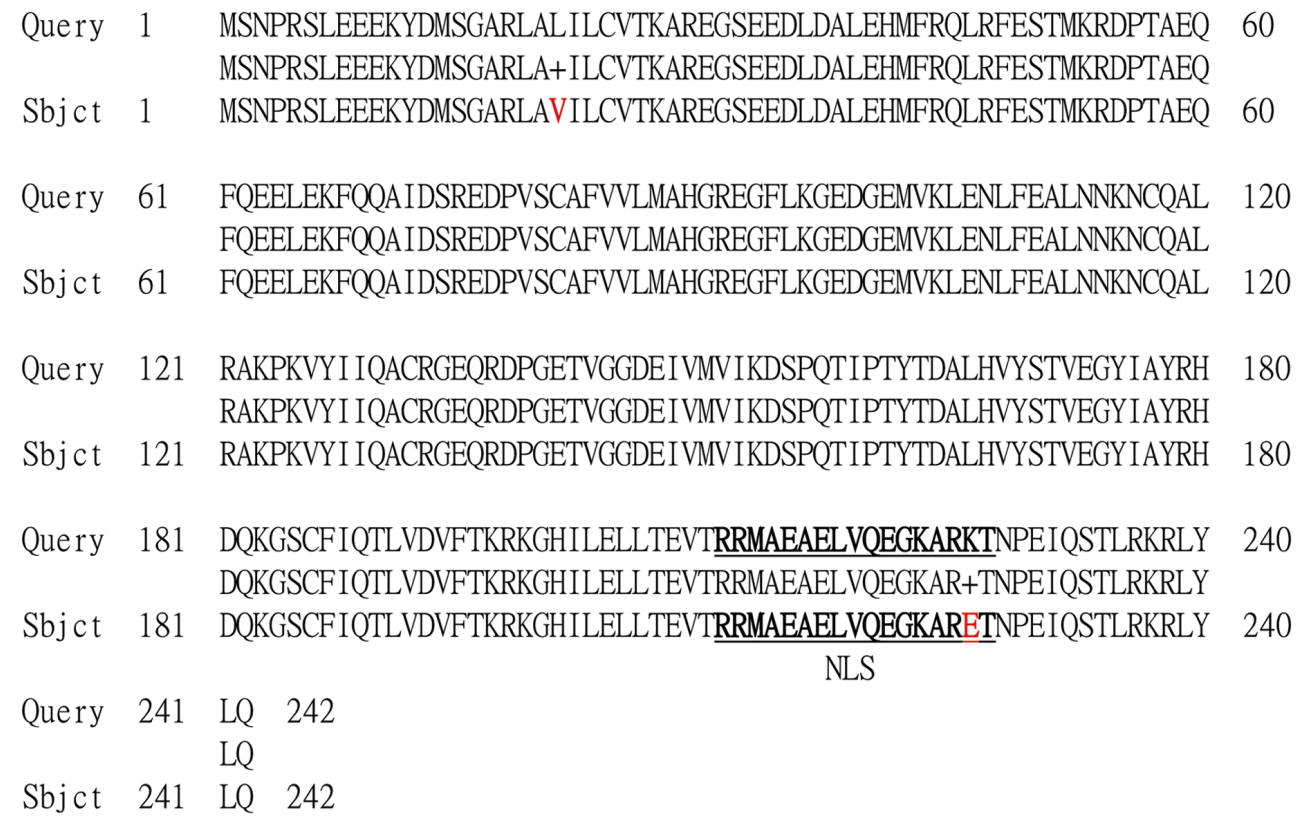

Figure 1. Expression and mutation of caspase-14 in human lung adenocarcinoma cells. (A) Expression of caspase-14 detected by RT-PCR. Expression of $\beta$-actin was used as a monitoring standard for the relative expression ratio of casp-14 mRNA. (B) The point mutation sites and the predicted nuclear localization signal of human caspase-14 in human lung adenocarcinoma cells. Compared to the published sequence, the sites of mutated amino acid residues in the caspase-14 are shown in red. Query, wild-type, NM_012114 777 bp mRNA linear PRI 16-AUG-2009 Homo sapiens caspase-14, apoptosis-related cysteine peptidase (CASP14), mRNA; Sbjct, mutant, EU262664 777 bp mRNA linear PRI 15-DEC-2007 Homo sapiens caspase-14 precursor (CASP14) mRNA, complete cds; NLS, nuclear localization signal shown in bold.

Table II. Probability of organelle distribution of various caspase-14 clones as predicted by a PSORT II program (http://psort. ims.u-tokyo.ac.jp/).

Clones of caspase-14

\begin{tabular}{|c|c|c|c|c|}
\hline Probability of organelle distribution & CASP- $14^{\mathrm{WT}}(\%)$ & Casp-14 $14^{\mathrm{Mu}}(\%)$ & Casp-14-N170 (\%) & Casp-14- $\Delta$ N50 (\%) \\
\hline Cytoplasmic & 65.2 & 65.2 & 60.9 & 78.3 \\
\hline Nuclear & 17.4 & 21.7 & 26.1 & 8.7 \\
\hline Mitochondrial & 8.7 & 4.3 & 4.3 & 4.3 \\
\hline Vacuolar & 4.3 & 4.3 & 4.3 & 4.3 \\
\hline Peroxisomal & 4.3 & 4.3 & 4.3 & 4.3 \\
\hline
\end{tabular}

WT, wild-type; Mu, mutation at Leu21 Val and Lys226Glu; Casp-14-N170 contained amino acids 1-170 of the human caspase-14, including the two hydrophobic segments. Casp-14- $\Delta$ N50 contained amino acids 51-242 of the human caspase-14 (deletion of the N-terminal 50 amino acid residues).

protein bands in both transfectants. Using immunoblotting, casp-14 was expressed 1 in H23, H226, H838, H2009, H2087 and A549 cells (Fig. 2B). Immunocytochemistry and fluorescence microscopy showed that casp-14 was present in the cytoplasm and in the nuclei (Fig. $2 \mathrm{C}$ and D). In pEGFP-casp-14 transfected cells, the EGFP-casp-14 was also detected in the cytoplasm and nuclei of the cells (Fig. 2E). Nucleolus, however, was devoid of casp-14, indicating 
A

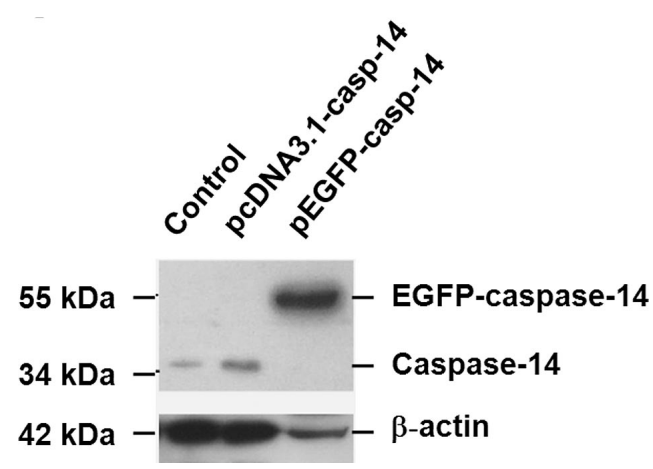

C

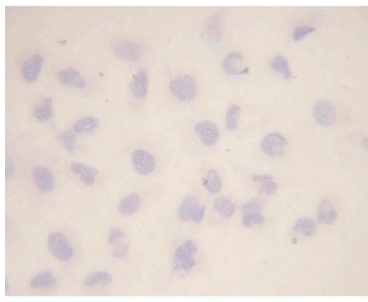

Negative control/H838

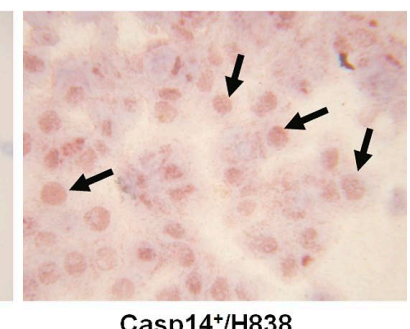

Casp14 $1 /$ H838
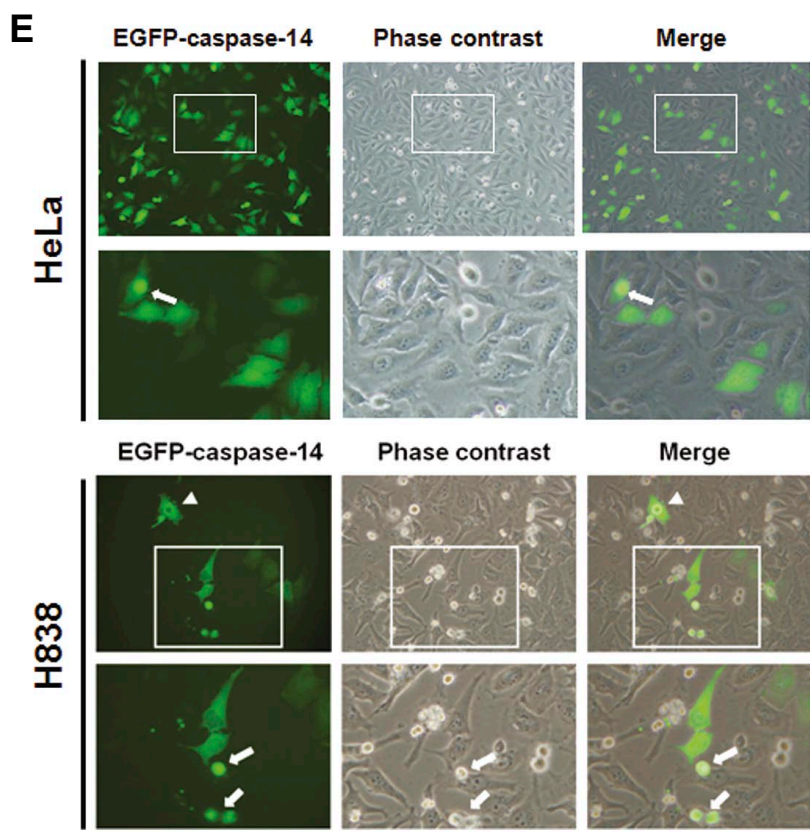

Phase contrast

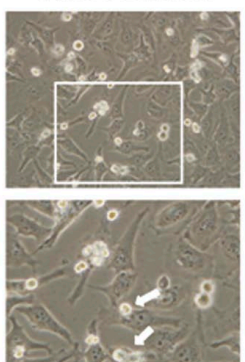

Merge

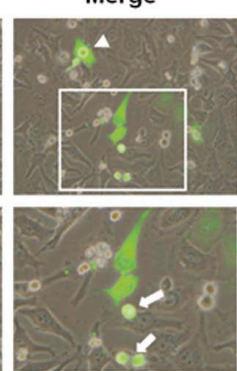

B

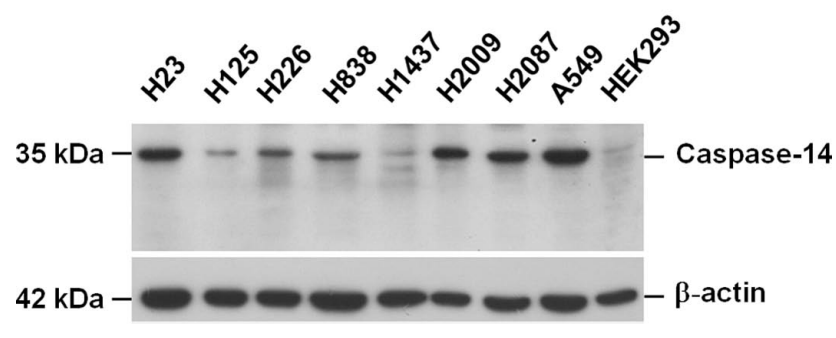

D

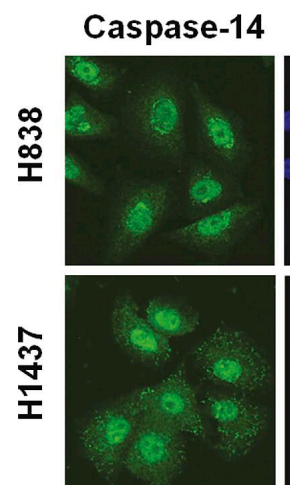

DAPI

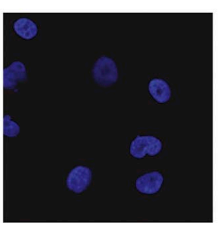

Merge
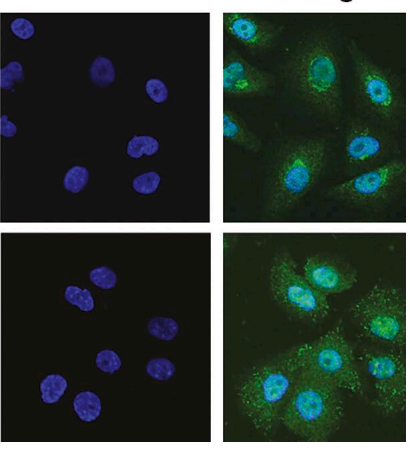

F

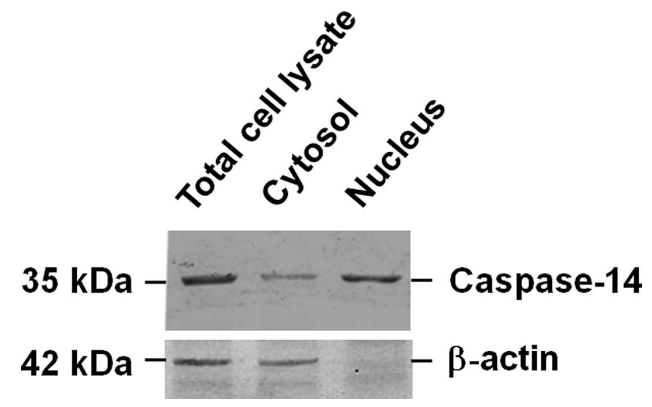

Figure 2. Characterization of monoclonal antibodies to caspase-14. (A) Immunoblotting revealed that monoclonal antibodies raised against recombinant casp-14 recognized a 35-kDa protein band in whole cell lysate from LADC cells or pcDNA3.1-casp-14 transfected LADC cells. In pEGFP-casp-14 transfected LADC cells, the recognized protein is 55-kDa. (B) Using immunoblotting, expression of caspase-14 was detected 1 in $\mathrm{H} 23$, $\mathrm{H} 226$, $\mathrm{H} 838$, $\mathrm{H} 2009$ and $\mathrm{H} 2087$ cells. (C) Immunocytochemical staining showed that compared to the negative control (the left panel), casp-14 was abundantly present in the cytoplasm and in the nuclei (the right panel, arrows). (D) Confocal immunofluorescence microscopy of two LADC cell lines, H838 and H1437. Caspase-14 was detected by antibodies labeled with fluorescein isothiocyanate (FITC). Nuclei were stained with fluorescent dye 4',6-diamidino-2-phenylindole (DAPI). A merged image of the first and the second columns confirms that caspase-14 is located in the nuclei. (E) In pEGFP-casp-14 transfected LADC cells, H838, and uterine cervical cancer cells, HeLa, the fluorescence protein was detected in the cytoplasm and the nuclei (white arrows) by a fluorescence microscopy. Nucleolus was devoid of pEGFP-casp-14 (white arrowhead). (F) Antibodies specific to caspase-14 recognized the protein both from the cytoplasmic and nuclear fractions as determined by an immunoblotting.

that casp-14 might not be involved in rRNA synthesis. Sub-organelle fractionation further showed that casp-14 was present in both the cytoplasm and the nucleus (Fig. 2F), confirming our microscopic observations. However, ectopic caspase-14 expression did not induce evident differentiation of LADC cells. 
A

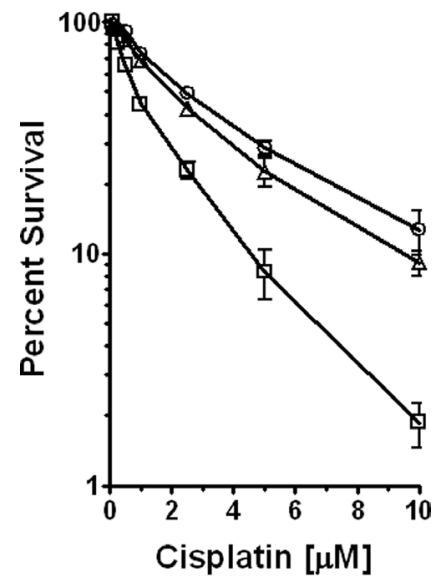

B2

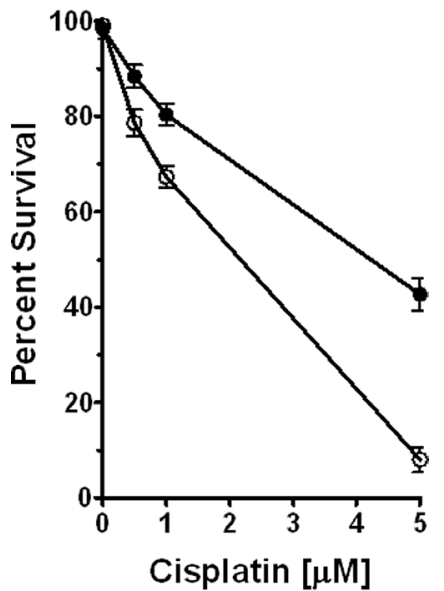

B1

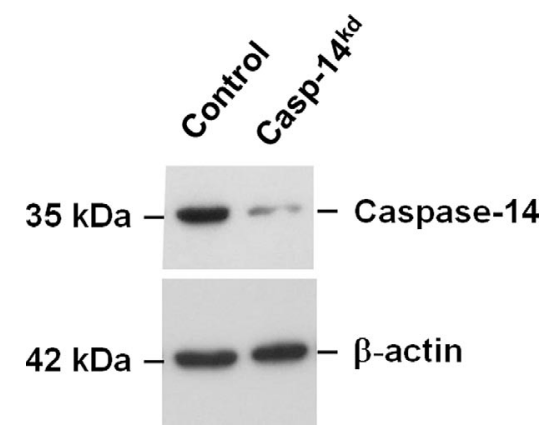

C

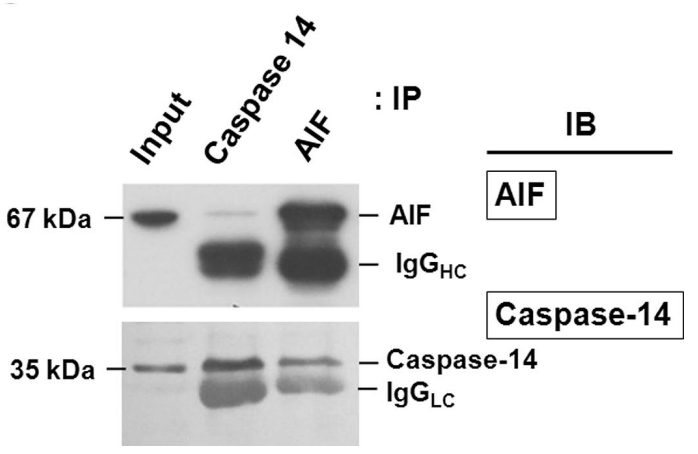

Figure 3. Effect of caspase-14 expression on cisplatin cytotoxicity. (A) Ectopic expression of caspase-14, either by transfection of pcDNA3.1-casp-14 (O) or

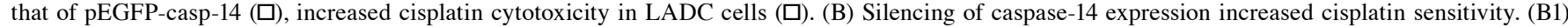
Using siRNA reduced $80 \%$ of caspase-14 expression in LADC cells [knockdown of caspase-14 (casp-14 ${ }^{\mathrm{kd}}$ )-specific expression]. (B2) Compared to the control LADC cells $(\bullet)$, casp-14 ${ }^{\mathrm{kd}}$ cells (O) are significantly more sensitive to cisplatin. (C) Casp-14 interacts with AIF. Monoclonal antibodies specific to caspase-14 precipitated caspase-14 (the lower panel) and AIF (the upper panel) from the whole cell lysate of H23 by protein G sepharose.

Increased caspase-14 reduces cisplatin sensitivity, probably via interaction with AIF. ALDC cells, which ectopically expressed casp-14 (transfection with pcDNA3.1-casp-14) or EGFP-casp-14 (transfection with pEGFP-casp-14), were more resistant to cisplatin (Fig. 3A). Using RNAi to silence casp-14 (casp-14 ${ }^{\mathrm{kd}}$ ) expression (Fig. 3B1), on the other hand, reduced cisplatin resistance (Fig. 3B2), supporting our expectations that expression of caspase-14 might increase drug resistance.

As noted previously, using a yeast two-hybrid assay, we had identified that AIF reacted with caspase-14 in cytoplasmic protein library. Moreover, antibodies specific to caspase-14 precipitated both caspase-14 and AIF from the whole cell lysate (Fig. 3C). Using deletion mutants to determine the specific binding segment of caspase-14, which was inserted into pGEX-4T-1/GST, GST-casp-14 and GST-casp-14-N152, but not GST-casp-14- $\Delta$ N152, pulled down AIF (Fig. 3D1). GST-casp-14-N152 contained amino acids 1-152 of the human caspase-14, including the two hydrophobic segments. GST-casp-14- $\Delta$ N152 contained amino acids $153-242$ of the human caspase-14 (deletion of the N-terminal 152 amino acid residues). Results of immunoprecipitation and protein pull-down assays confirmed our data of yeast two hybrid assays that caspase-14 interacted with AIF. Results of pulldown assay further showed that the ratio between AIF and caspase-14 interaction was about 1 to 8 , i.e., 1 AIF interacted with 8 caspase-14. Point mutation in the hydrophobic segments reduced GST pull-down levels of AIF (Fig. 3D2) indicated that interaction between caspase-14 and AIF could be hydrophobic. Using a web program PyMOL (http:// www.pymol.org) for predicting a 3D structure showed that caspase-14 contained $5 \alpha$-helices and $6 \beta$-sheets (Fig. 3E). The first hydrophobic segment (amino acid residues 19-25) and the second hydrophobic segment (amino acid residues 79-88) could form 2 parallel $\beta$-sheets, which with the neighboring $\alpha$-helices could constitute a binding pocket.

Expression of caspase-14 in LADC correlated with increase of tumor recurrence and decrease of patient's survival. Using immunoblotting, while most of the caspase-14 detected in the non-tumor lung tissues (NTLT) was 35-kDa, two caspase-14positive bands, 35- and 37-kDa, were identified in the LADC specimens (Fig. 4A). Because the molecular shift is minor and based on our previous experience (26), we proposed that the $37-\mathrm{kDa}$ band was a phosphorylated form of caspase-14. 
D1

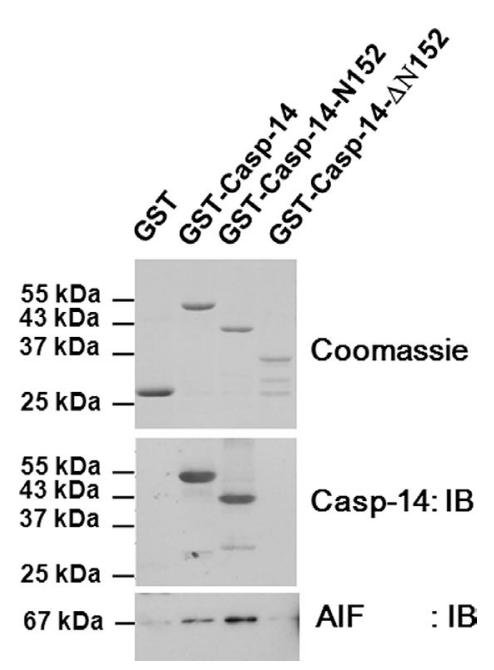

E

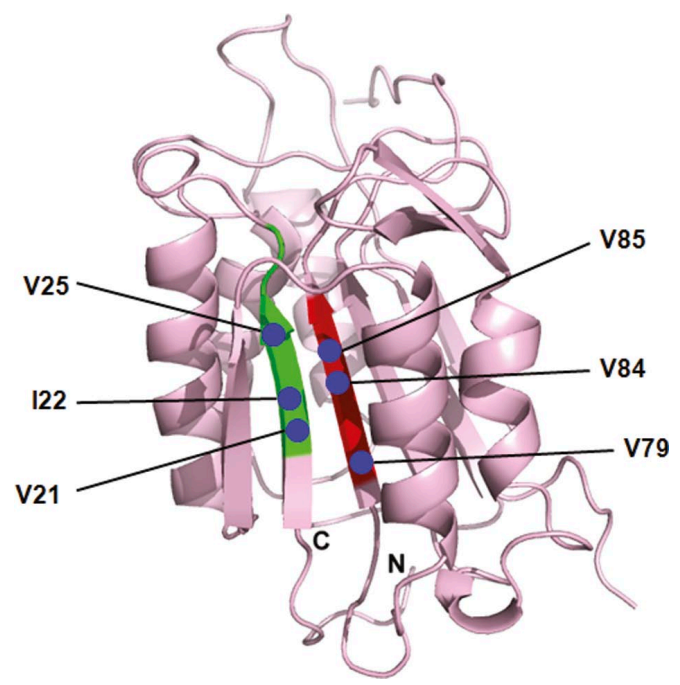

The tissue lysate of LADC was treated with calf intestinal alkaline phosphatase (CIP) before immunoblotting, and the results showed a reduction in signals of the $37-\mathrm{kDa}$ protein band (Fig. 4B), indicating that the $37-\mathrm{kDa}$ protein was a phosphorylated caspase-14. We then used a NetPhos program (http://www.cbs.dtu.dk/services/NetPhos/) to predict for phosphorylation sites, and a NetPhosK program (http://www.cbs. dtu.dk/services/NetPhosK/) to predict for the specific kinase, the results showed that the most probable kinase was protein kinase C (PKC) at ${ }^{201} \mathrm{Thr}$ for caspase-14. However, we did not detect any LADC cell line that is expressing the $37-\mathrm{kDa}$ caspase-14. We therefore tested our antibodies on psoriatic lesions by immunohistochemistry (Fig. 4C1), as well as psoriatic specimens and mouse organs by immunoblotting (Fig. 4C2). The results were similar to previous reports $(14,22)$.

Using immunohistochemistry, caspase-14 was detected in some of type II pneumocytes of NTLT (Fig. 4D1). However, caspase-14 signals were detected in $88(82.2 \%)$ of LADC pathological samples. The signal was mainly located in the cancer cells (Fig. 4D2), but not in the neighboring inflammatory cells. In 80 (90.9\%) of the pathological specimens caspase-14 was identified in the nuclei (Fig. 4D3). Caspase-14 expression was also detected in $83.9 \%$ (47/56) of metastaticlymph nodes (data not shown). In the presence of recombinant
D2

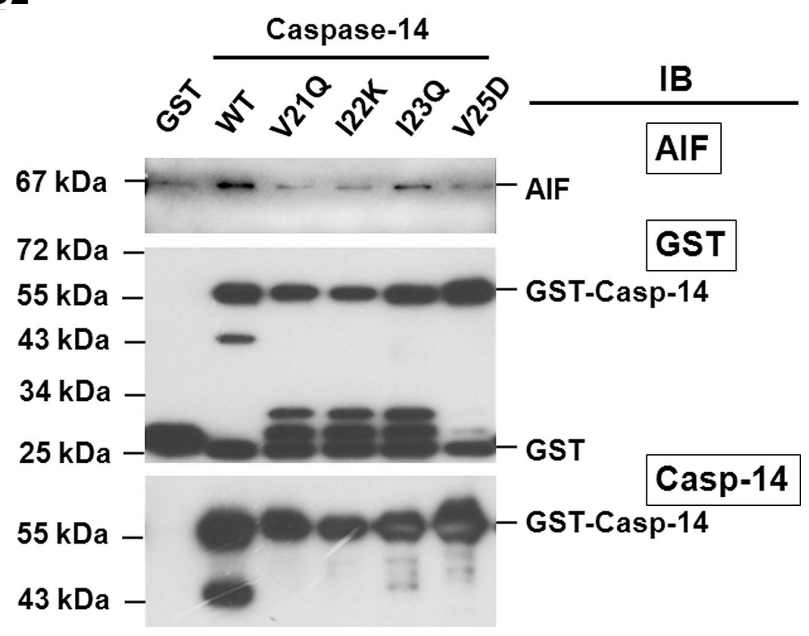

Figure 3. (D) using GST-pull down assay to determine the interaction between caspase-14 and AIF. (D1) Full-length and partial fragments of caspase-14 were inserted into pGEX-4T-1/GST (upper panel). GST-casp-14 contained the full-length of caspase-14 gene, while deletion mutants contained only segments of caspase-14, i.e., GST-casp-14-N152 contained amino acids $1-152$, and GST-casp-14- $\Delta$ N152 contained amino acids 153 242 (deletion of the N-terminal 152 amino acid residues) of the human caspase-14 gene (central panel). GST-casp-14 and GST-casp-14-N152, but not GST-casp-14- $\Delta$ N152, could pull down AIF (bottom panel) as determined by immunoblotting. (D2) Point mutation to change an aliphatic into polar side-chain (at V21, I22, I23 or V25) of amino acid residues markedly reduced GST pull-down levels of AIF suggested that the interaction between caspase-14 and AIF was hydrophobic. (E) Using a web program PyMOL (http://www.pymol.org) to predict a 3D protein structure showed that caspase-14 contained $5 \alpha$-helices and $6 \beta$-sheets. The first hydrophobic segment (amino acid residues 19-25) and the second hydrophobic segment (amino acid residues 79-88) formed 2 parallel $\beta$-sheets, which with the neighboring $\alpha$-helices could constitute an interaction pocket.

casp-14, the immunostaining signals reduced markedly or completely abolished (data not shown). Statistical analysis showed that overexpression of caspase-14 in tumors correlated with tumor stage, cigarette smoking, lymphovascular involvement and cell differentiation (Table III), suggesting that expression of caspase-14 was associated with tumor cell growth and metastatic potential of LADC cells. Interestingly, among the 88 patients who had high levels of caspase-14, $38(43.2 \%)$ patients had tumor recurrence during follow-up examination. Among the 19 patients who had low levels of caspase-14, four had tumor recurrence (21.0\%). All 42 patients who had recurrence developed tumors within 24 months after operation. The risk of recurrence for patients with high levels of casp-14 was 2.31-fold higher than that for patients with low levels of casp-14 $(\mathrm{p}=0.029)$. Survival of patients with low casp-14 levels was significantly better than that of patients with high casp-14 levels (Fig. 4E1). The hazard ratio between these two groups was 2.323 , and the difference in cumulative survival was significant $(\mathrm{p}=0.0022)$. Multivariate analysis, however, did not show a significant statistical difference in casp-14 expression between the two groups ( $\mathrm{p}=0.0821)$. When casp-14+ group was subcategorized into nucleus-positive (nCasp-14 $4^{+}$and cytoplasm-positive (cytCasp-14+) groups, though a significant difference was found in the survival among the three groups $(\mathrm{p}=0.0302)$, no significant difference 
A
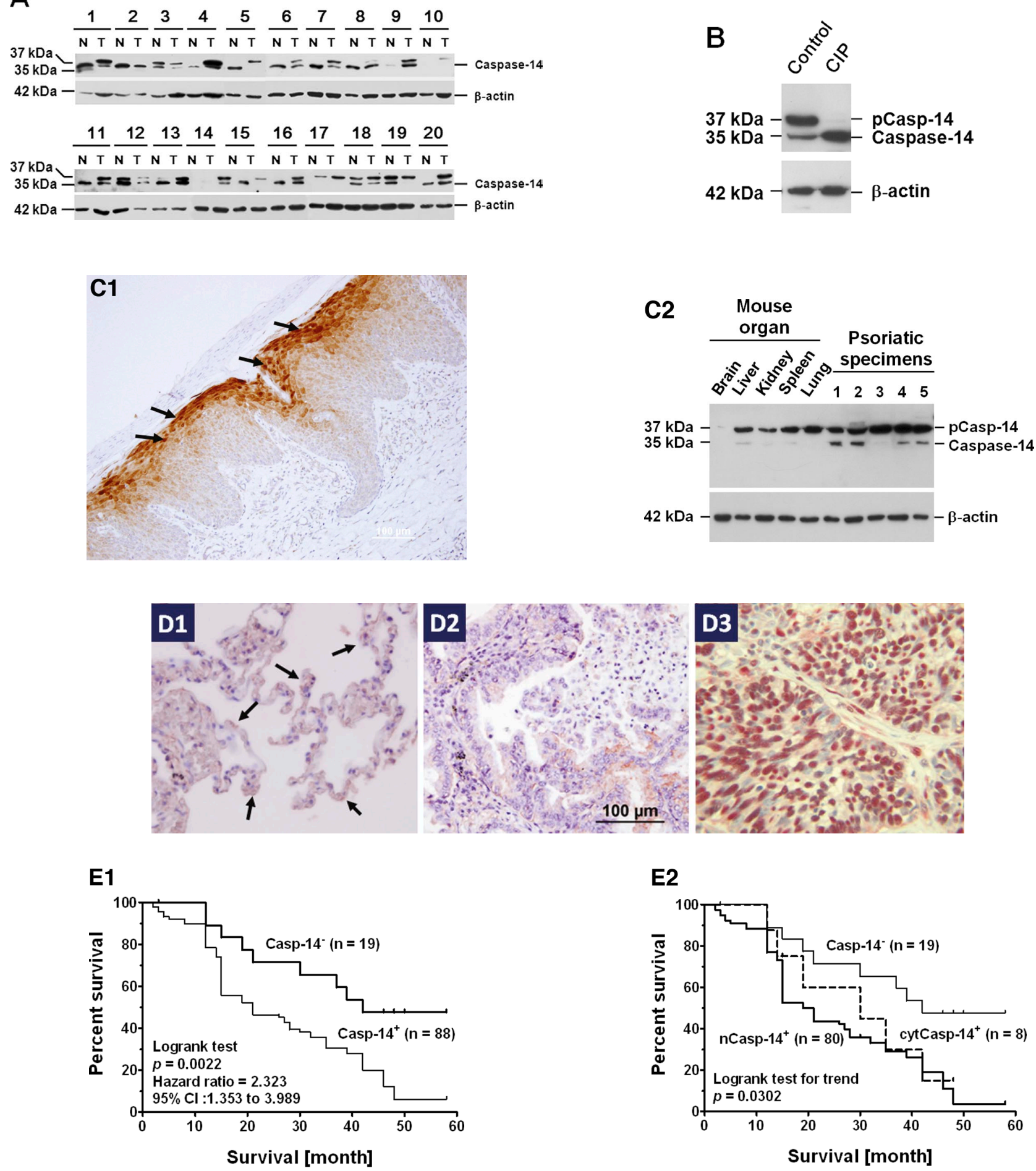

Figure 4. Correlation between casp-14 expression and survival in LADC patients. (A) Expression of casp-14 was determined by immunoblotting. Expression of $\beta$-actin was used as a monitoring standard for the relative expression of caspase-14. While the $35-\mathrm{kDa}$ caspase-14 was mostly detected in the NTLT, two prominent caspase-14-positive protein bands, 35- and 37-kDa, were identified in the LADC specimens. N, non-tumor lung tissues; NTLT' T, tumor fraction of surgical resections. (B) Pooled tissue lysate (75 $\mu \mathrm{g}$ ) of LADC was treated with calf intestinal alkaline phosphatase (CIP) before immunoblotting. CIP treatment reduced signals of 37-kDa band, suggesting that the 37-kDa caspase-14 could be a phosphorylated protein (pCasp-14). (C) Expression of caspase-14 in the pathological specimen of psoriatic lesions. (C1) Expression of caspase-14 was detected by an immunohistochemical staining (as brown precipitates using DAB for chromogens). Expression of caspase-14 was detected in the cytoplasm and the nuclei of aberrantly proliferated epidermal cells (arrows), but not in the cornified layer. (C2) Expression of caspase-14 in mouse tissues and pathological specimen of psoriatic lesions as determined by immunoblotting. (D) Representative examples of caspase-14 expression in pathological specimens as detected by immunohistochemical staining (as crimson precipitates). Compared to (D1) the NTLT, in which the positively stained cells were mostly type II pneumocytes (arrows), expression of caspase-14 was detected in (D2) the cytoplasm and (D3) the nuclei of LADC tumor cells. (E) Comparison of Kaplan-Meier product limit estimates of survival analysis in LADC patients. (D1) Patients were divided into two groups based on expression of casp-14. Survival difference between the two groups was compared by a log-rank test. $\mathrm{p}=0.0022$; (D2) to determine the effect of nuclear casp-14 on survival, the casp-14+ group was subcategorized into nucleus-positive (nCasp- $14^{+}$) and cytoplasm-positive

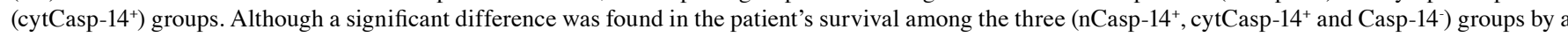
log-rank test for trend $(\mathrm{p}=0.0302)$, no significant difference was detected between $\mathrm{nCasp}-14^{+}$and cytCasp-14+ groups $(\mathrm{p}=0.47)$ or that between casp-14 and cytCasp- $14^{+}$groups $(\mathrm{p}=0.147)$. 
Table III. Correlation of casp-14 expression with clinicopathological parameters in patients with LADC.

\begin{tabular}{|c|c|c|c|c|c|}
\hline \multirow[b]{2}{*}{ Parameter } & \multirow[b]{2}{*}{ No. } & \multicolumn{2}{|c|}{ Expression of casp-14 } & \multicolumn{2}{|c|}{ P-value } \\
\hline & & High $(n=88)$ & Low $(n=19)$ & Univariate & Multivariate \\
\hline \multicolumn{6}{|l|}{ Gender } \\
\hline Male & 84 & 72 & 12 & $0.073^{\mathrm{a}}$ & \\
\hline Female & 23 & 16 & 7 & & \\
\hline \multicolumn{6}{|c|}{ Cigarette smoking } \\
\hline Smoker & 78 & 68 & 10 & $0.028^{\mathrm{a}}$ & 0.057 \\
\hline Non-smoker & 29 & 20 & 9 & & \\
\hline \multicolumn{6}{|l|}{ Stage } \\
\hline $\mathrm{I}$ & 26 & 18 & 8 & $0.002^{\mathrm{b}}$ & 0.018 \\
\hline II & 31 & 22 & 9 & & \\
\hline III & 50 & 48 & 2 & & \\
\hline \multicolumn{6}{|c|}{ Cell differentiation } \\
\hline Well & 17 & 10 & 7 & $0.019^{\mathrm{b}}$ & 0.076 \\
\hline Moderate & 60 & 51 & 9 & & \\
\hline Poor & 30 & 27 & 3 & & \\
\hline \multicolumn{6}{|c|}{ Lymphovascular invasion } \\
\hline Positive & 81 & 69 & 12 & $0.029^{\mathrm{a}}$ & 0.060 \\
\hline Negative & 26 & 19 & 7 & & \\
\hline
\end{tabular}

${ }^{a}$ Two-sided $\mathrm{p}$-value determined by $\chi^{2}$ test; ${ }^{\text {b}}$ Two-sided $\mathrm{p}$-value determined by Fisher's exact test.

was found between the nCasp- $14^{+}$and the cytCasp- $14^{+}$groups $(\mathrm{p}=0.47)$ or that between the casp-14- and the cytCasp-14 ${ }^{+}$ groups $(\mathrm{p}=0.147)$ (Fig. 4E2). The outcome could be due to the low sample number of cytoplasm-positive cases.

\section{Discussion}

The data presented above showed that overexpression of casp-14 was frequently detected in pathological specimens of LADC. Moreover, casp-14 expression is significantly correlated with higher incidence of the early tumor recurrence and the increased drug resistance, which ultimately reflects in the poor survival of LADC patients. In the in vitro experiments, casp-14 bound the apoptosis-inducing factor (AIF). The increased casp-14 level therefore correlated with reduced cisplatin sensitivity. These results support our hypothesis that casp-14 is an anti-apoptotic protein, probably, targeting at AIF in LADC cells.

Caspases and AIF have in recent years emerged as novel targets for anticancer drugs, including cisplatin, adriamycin, camptothecin and etoposide $(3,12)$. Current evidence suggests that drugs induce destabilization of mitochondrial membrane integrity, procaspase activation and nuclear translocation of AIF, which provokes DNA fragmentation, micronuclei formation and cell death (8). Maintaining mitochondrial membrane integrity and interfering caspase activation as well as nuclear translocation of AIF could then reduce drug-induced apoptosis. As noted above, using AIF as bait in a yeast two hybrid assay, microsomal GST,
hHR23A and casp-14 were identified in the protein libraries, which interacted with AIF. Moreover, unlike other caspases, casp-14 is not cleaved at Asp residue of the linker region when cells were exposed to genotoxic stress. Using immunoprecipitation and protein pull-down assays, we showed that AIF interacted with casp-14. By demonstrating that ectopic expression of casp-14 decreased cisplatin-mediated cell killing, and silencing of casp-14 expression increased cisplatin sensitivity, our data showed that casp-14 could inhibit drug-induced cell death, and suggested that the cytoprotection effect of casp-14 might possibly be via its binding to AIF and the consequent interferece of AIF nuclear translocation.

In addition to AIF, nuclear level of other apoptosis-associated mitochondrial proteins, such as apoptotic protease activating factor 1 (Apaf-1), B cell lymphoma protein 2 (Bcl-2), $\mathrm{BH} 3$-interacting death domain protein (BID), and $\mathrm{Bcl}-2$ $19 \mathrm{kDa}$ interacting protein 3 (BNIP3), increases following genotoxic stresses, including $U V$ irradiation, ionizing radiation and chemotherapeutics (26-29). Recent studies show that when these proteins are present in the nuclei, they are involved in the cell cycle progression and DNA repair, the processes that are mediated by $\mathrm{p} 53$, checkpoint (Chk)-1, Chk-2, ataxia telangiectasia mutated (ATM) as well as ATM and rad3-related (ATR) kinases (30). Nuclear presence of these proteins increases genomic instability as well as resistance to irradiation and anticancer drugs $(29,31)$, suggesting that the nuclear presence of these proteins is vital for cell survival. 
Nuclear casp-14 has been detected in the stratum spinosum of normal skin, psoriatic lesions and epithelial malignancies $(17,18,22)$. However, casp-14 is not detected in basal and cornified layers of normal skin or in basal layer and parakeratotic abrasion of psoriasis. Since skin cells in basal layer and parakeratotic abrasion are undifferentiated cells, the results suggested that the protein could be involved in the differentiation of squamous cells $(16,22)$. The role of nuclear casp-14 in cell differentiation, however, was not clear. By showing that knockout of casp-14 gene increases UVB sensitivity, Denecker et al suggest that casp-14 is essential for reducing dermal accumulation of the cyclobutane pyrimidine dimers (CPD), and for the increasing p53-related cell survival (16). Involvement of casp-14 in these nuclear events, however, was not elucidated. It is worth noting that following $U V$ damage xeroderma pigmentosum complementation group C (XPC) protein and human homolog of yeast $\operatorname{Rad} 23$ protein B (hHR23B) are promptly recruited to the nucleus (32). Inside nucleus, hHR23B increases p53 accumulation and inhibits cell cycle progression, while XPC accelerates DNA repair and reduces DNA damage-associated apoptosis. Cooperation between XPC and hHR23B renders cells resistant to chemotherapeutics and irradiation (32). A recent report showed that increased nuclear phosphorylation level of DRP1, which was accompanied by the nuclear accumulation of hHR23A under hypoxic condition, was vital for the protection of nucleoli and reduction of cisplatin sensitivity (33). Taken together, our results clearly show that casp-14, either cytoplasmic or nuclear, plays an imperative role in protecting cells from genotoxic damages, possibly by interfering nuclear translocation of AIF and the subsequent binding of AIF to genomic DNA. The essence of nuclear casp-14 is yet to be determined.

Interestingly, oxidative stress increases nuclear translocation of focal adhesion kinase (FAK), an integrin- and hepatocyte growth factor (HGF)-associated tyrosine kinase which is normally located on plasma membrane, to modulate muscle differentiation as well $(34,35)$. Knockdown of FAK expression increased AIF expression and reduced cisplatin resistance of mouse embryonic fibroblasts $(12,19)$. These results correspond well with our current findings, indicating that under unfavorable growth conditions, such as nutrient depletion or hypoxia, the cells may initiate emergency nuclear transport of vital proteins, such as FAK, DRP1 and casp-14, and activate expression of cyto-defensive genes to protect genomic DNA from self destruction $(12,19,26,35)$. The nuclear events, however, are yet to be elucidated.

In conclusion, immunohistochemistry revealed overexpression of casp-14 in lung adenocarcinomas. Pathological results show that casp-14 expression is associated with poor prognosis. In vitro, expression of casp-14 increased cisplatin resistance in LADC cells. By showing that casp-14 and AIF interacted with each other, and that silencing of casp-14 decreased cisplatin resistance, our results suggest that casp-14 may play an important role in interfering nuclear function of AIF and increase drug resistance of LADC cells.

\section{Acknowledgements}

RNAi for silencing casp-14 gene expression was obtained from the National RNAi Core Facility in the Institute of
Molecular Biology/Genomic Research Center, Academia Sinica, Taipei, Taiwan, supported by the National Research Program for Genomic Medicine Grants of NSC (NSC 97-3112-B-001-016). This study was supported in part by the Comprehensive Academic Promotion Projects (NCHU 985005, from Department of Education, Executive Yuan, Republic of China, to K.C.C.).

\section{References}

1. Taylor RC, Cullen SP and Martin SJ: Apoptosis: controlled demolition at the cellular level. Nat Rev Mol Cell Biol 9: 231-241, 2008.

2. Clarke PG: Developmental cell death: morphological diversity and multiple mechanisms. Anat Embryol 181: 195-213, 1990

3. Gozuacik D and Kimchi A: Autophagy as a cell death and tumor suppressor mechanism. Oncogene 23: 2891-2906, 2004.

4. Bras M, Yuste VJ, Roué G, et al: Drp1 mediates caspaseindependent type III cell death in normal and leukemic cells. Mol Cell Biol 27: 7073-7088, 2007.

5. Lorenzo HK and Susin SA: Mitochondrial effectors in caspaseindependent cell death. FEBS Lett 557: 14-20, 2004.

6. Jaattela M and Tschopp J: Caspase-independent cell death in T lymphocytes. Nat Immunol 4: 416-423, 2003.

7. Susin SA, Lorenzo HK, Zamzami N, et al: Molecular characterization of mitochondrial apoptosis-inducing factor. Nature 397: 441-446, 1999.

8. Daugas E, Susin SA, Zamzami N, et al: Mitochondrio-nuclear translocation of AIF in apoptosis and necrosis. FASEB J 14: 729-739, 2000.

9. Arnoult A, Gaume B, Karbowski M, Sharpe JC, Cecconi F and Youle RJ: Mitochondrial release of AIF and EndoG requires caspase activation downstream of Bax/Bak-mediated permeabilization. EMBO J 22: 4385-4399, 2003.

10. Wu J, Harris NL and Inge TH: Nuclear factor-kappa B and apoptosis inducing factor activation by doxorubicin analog WP744 in SH-SY5Y neuroblastoma cells. J Surg Res 122: 231-239, 2004.

11. Rodríguez-Hernández A, Brea-Calvo G, Fernández-Ayala DJ, Cordero M, Navas P and Sánchez-Alcázar JA: Nuclear caspase-3 and caspase-7 activation, and poly(ADP-ribose) polymerase cleavage are early events in camptothecin-induced apoptosis. Apoptosis 11: 131-139, 2006.

12. Chen JT, Huang CY, Chiang YY, et al: HGF increases cisplatin resistance via down-regulation of AIF in lung cancer cells. Am J Respir Cell Mol Biol 38: 559-565, 2008.

13. Chook YM and Blobel G: Karyopherins and nuclear import. Curr Opin Struct Biol 11: 703-715, 2001.

14. Van de Craen M, van Loo G, Pype S, et al: Identification of a new caspase homologue: caspase-14. Cell Death Differ 5: 838-846, 1998.

15. Chien AJ, Presland RB and Kuechle MK: Processing of native caspase-14 occurs at an atypical cleavage site in normal epidermal differentiation. Biochem Biophys Res Commun 296: 911-917, 2002.

16. Denecker G, Hoste E, Gilbert B, et al: Caspase-14 protects against epidermal UVB photodamage and water loss. Nat Cell Biol 9: 666-674, 2007.

17. Koenig U, Sommergruber W and Lippens S: Aberrant expression of caspase-14 in epithelial tumors. Biochem Biophys Res Commun 335: 309-313, 2005.

18. Krajewska M, Kim H, Shin E, et al: Tumor-associated alterations in caspase-14 expression in epithelial malignancies. Clin Cancer Res 11: 5462-5471, 2005.

19. Chen JT, Lin TS, Chow KC, et al: Cigarette smoking induces overexpression of HGF in type II pneumocytes and lung cancer cells. Am J Respir Cell Mol Biol 34: 264-273, 2006.

20. Brownson RC, Alavanja MC, Hock ET and Loy TS: Passive smoking and lung cancer in non-smoking women. Am J Public Health 82: 1525-1530, 1992.

21. Detterbeck FC, Boffa DJ and Tanoue LT: The new lung cancer staging system. Chest 136: 260-271, 2009.

22. Lippens S, Kockx M, Knaapen M, et al: Epidermal differentiation does not involve the pro-apoptotic executioner caspases, but is associated with caspase-14 induction and processing. Cell Death Differ 7: 1218-1224, 2000. 
23. Remmele W and Schicketanz KH: Immunohistochemical determination of estrogen and progesterone receptor content in human breast cancer. Computer-assisted image analysis (QIC score) vs. subjective grading (IRS). Pathol Res Pract 189: 862-866, 1993.

24. Kaplan EL and Meier P: Non-parametric estimation from incomplete observations. J Am Stat Assoc 53: 457-481, 1958.

25. Mantel N: Evaluation of survival data and two new rank order statistics arising in its consideration. Cancer Chemother Rep 50: 163-170, 1966.

26. Kamer I, Sarig RH, Zaltsman Y, et al: Proapoptotic BID is an ATM effector in the DNA-damage response. Cell 122: 593-603, 2005.

27. Zinkel SS, Hurov KE, Ong C, Abtahi FM, Gross A and Korsmeyer SJ: A role for proapoptotic BID in the DNA-damage response. Cell 122: 579-591, 2005.

28. Burton TR, Henson ES, Baijal P, Eisenstat DD and Gibson SB: The pro-cell death Bcl-2 family member, BNIP3, is localized to the nucleus of human glial cells: Implications for glioblastoma multiforme tumor cell survival under hypoxia. Int J Cancer 118: 1660-1669, 2006.
29. Zermati Y, Mouhamad S, Stergiou L, et al: Non-apoptotic role for Apaf-1 in the DNA damage checkpoint. Mol Cell 28: 624-637, 2007

30. Bartek J, Bartkova J and Lukas J: DNA damage signalling guards against activated oncogenes and tumour progression. Oncogene 26: 7773-7779, 2007.

31. Kaur M, Pop M, Shi D, Brignone C and Grossman SR: hHR23B is required for genotoxic-specific activation of p53 and apoptosis. Oncogene 26: 1231-1237, 2007.

32. Dominguez-Brauer C, Chen YJ, Brauer PM, Pimkina J and Raychaudhuri P: ARF stimulates XPC to trigger nucleotide excision repair by regulating the repressor complex of E2F4. EMBO Rep 10: 1036-1042, 2009.

33. Chiang YY, Chen SL, Hsiao YT, et al: Nuclear expression of dynamin-related protein 1 in lung adenocarcinomas. Mod Pathol 22: 1139-1150, 2009.

34. Luo SW, Zhang C, Zhang B, et al: Regulation of heterochromatin remodeling and myogenin expression during muscle differentiation by FAK interaction with MBD2. EMBO J 28: 2568-2582, 2009.

35. Lim ST, Chen XL, Lim Y, et al: Nuclear FAK promotes cell proliferation and survival through FERM-enhanced p53 degradation. Mol Cell 29: 9-22, 2008. 